\title{
A Sensitive Fluorescent Method for the Detection of Glycoproteins in Polyacrylamide Gels ${ }^{1}$
}

\author{
Allen E. Eckhardt, Colleen E. Hayes, and Irwin J. Goldstein \\ Department of Biological Chemistry, University of Michigan, Ann Arbor, Michigan 48109
}

Received November 21, 1975; accepted January 22, 1976

\begin{abstract}
A sensitive method is described for the detection of glycoproteins after polyacrylamide gel electrophoresis. Dansyl hydrazine is condensed with aldehydic functions generated by periodate oxidation of protein bound carbohydrate. The resulting hydrazones are reduced to the stable hydrazine derivatives with sodium borohydride. After destaining, the fluorescent-labeled glycoproteins are visualized with a long-range uv light source. The dansyl hydrazine staining technique was shown to detect less than $40 \mathrm{ng}$ of carbohydrate bound to Bandeiraea simplicifolia lectin.
\end{abstract}

The principle methods for the detection of glycoproteins in polyacrylamide gels are the periodic acid-Schiff stain (PAS) $(1,2,4)$ and the Alcian blue stain (3). Both of these procedures involve the fixation of the glycoproteins in the gel followed by oxidation of sugar residues with periodic acid generating aldehydic functions, reduction of the excess periodate with sodium metabisulfite and staining with either basic fuchsin or Alcian blue. The minimum amount of protein-bound carbohydrate which can be detected with these methods is in the range of $2-3 \mu \mathrm{g}$ (4).

The fluorescent reagent 1-dimethylaminonaphthalene-5-sulfonylhydrazine (dansyl hydrazine) has been used for the detection and quantitation of carbonyl containing compounds such as keto steroids (5). We report here the use of this reagent as a sensitive fluorescent stain for the visualization of glycoproteins following polyacrylamide gel electrophoresis. The glycoproteins are subjected to periodate oxidation and the resulting adehydes condensed with dansyl hydrazine to form hydrazones. The latter are reduced to stable hydrazine derivatives with $\mathrm{NaBH}_{4}$.

\section{MATERIALS AND METHODS}

Reagents. Reagents were from the following sources: dansyl hydrazine, used without further purification, Pierce Chemical Co.; paraperiodic acid, G. Frederick Smith Chemical Corp.; sodium borohydride, Metal Hydrides Inc.; bovine serum albumin, Pentex; Ovalbumin (two times crystallized), Nutritional Biochemicals; chymotrypsinogen (5 times crystallized), Worthington Biochemicals. Concanavalin A (6), Bandeiraea

${ }^{1}$ This study was supported by National Institutes of Health Grant AM 10171 and Training Grant GM 00187. 
simplicifolia lectin (7) and Phaseolus lunatus lectin (8) were prepared as previously described.

Protein determination. Proteins were dialyzed against $0.15 \mathrm{M} \mathrm{NaCl}$ for $24 \mathrm{hr}$ and the concentration determined by the method of Lowry et al. (9) using bovine serum albumin as standard.

Electrophoresis. Polyacrylamide gel electrophoresis was performed according to the method described by Reisfeld et al. (10). The gels were polymerized in $0.5 \times 7 \mathrm{~cm}$ tubes which had been precoated with $2 \%$ aqueous Photo-flo 200 and dried. Due to its fluorescence at $550 \mathrm{~nm}$, riboflavin was omitted from the stacking gel and replaced by ammonium persulfate as the free radical producing catalyst. Samples to be electrophoresed were adjusted to $10 \%$ glycerol $(\mathrm{v} / \mathrm{v})$ and $0.005 \%$ methylene green $(w / v)$, layered on the stacking gel and electrophoresed at $4 \mathrm{~mA}$ per gel for $1.5 \mathrm{hr}$. Immediately following electrophoresis, the gels were removed and stained for protein, using Coomassie brilliant blue, or for carbohydrate. using the PAS stain, Alcian blue, or dansyl hydrazine.

Protein stain. Gels were stained for protein with $0.05 \%$ Coomassie brilliant blue (w/v) in $10 \%$ aqueous acetic acid, overnight, followed by destaining against several changes of $7 \%$ aqueous acetic acid.

$P A S$ stain. Gels were fixed overnight in an aqueous solution containing $40 \%$ ethanol and $5 \%$ glacial acetic acid by volume and then stained for sugar with PAS according to the method of Segrest et al. (1).

Alcian blue stain. Gels were fixed overnight in an aqueous solution containing $40 \%$ ethanol, $5 \%$ glacial acetic acid by volume, and then stained for carbohydrate with Alcian blue $8 \mathrm{GX}$ according to the method of Wardi et al. (3).

Dansyl hydrazine stain. Gels, fixed overnight in an aqueous solution containing $40 \%$ ethanol, $5 \%$ glacial acetic acid by volume, were oxidized with periodic acid at room temperature according to the method of Segrest et al. (1). To each gel in a test tube was added approx $20 \mathrm{ml} 0.7 \%$ paraperiodic acid $(\mathrm{w} / \mathrm{v})$ in $5 \%$ aqueous acetic acid by volume and the solution allowed to stand for $2 \mathrm{hr}$. The gels were rinsed with distilled water and treated with $0.5 \%$ sodium metabisulfite in $5 \%$ aqueous acetic acid for 1 to $1.5 \mathrm{hr}$ until colorless, in order to destroy excess periodic acid. The gels were rinsed several times with distilled water and placed in $10 \times 75 \mathrm{~mm}$ test tubes. To each tube was added equal volumes of acidic DMSO solution $(0.60 \mathrm{ml}$ concentrated hydrochloric acid per liter DMSO) and freshly prepared dansyl hydrazine solution (dansyl hydrazine, $2 \mathrm{mg} / \mathrm{ml}$ DMSO). The tubes were stoppered (rubber stoppers), mixed and incubated for $2 \mathrm{hr}$ in a water bath maintained at $60^{\circ} \mathrm{C}$. The dansyl hydrazine-DMSO solution was poured off and $5 \mathrm{ml}$ of a solution of $\mathrm{NaBH}_{4}$ in DMSO $(0.2 \mathrm{mg} \mathrm{NaBH} / \mathrm{ml}$ DMSO) was added to each tube and incubated for $30 \mathrm{~min}$ at room temperature. The gels were rinsed with distilled $\mathrm{H}_{2} \mathrm{O}$ and destained overnight in $1 \%$ aqueous glacial acetic acid $(\mathrm{v} / \mathrm{v})$ with several changes. Destaining was complete when the gel background was colorless when illuminated with uv light. 

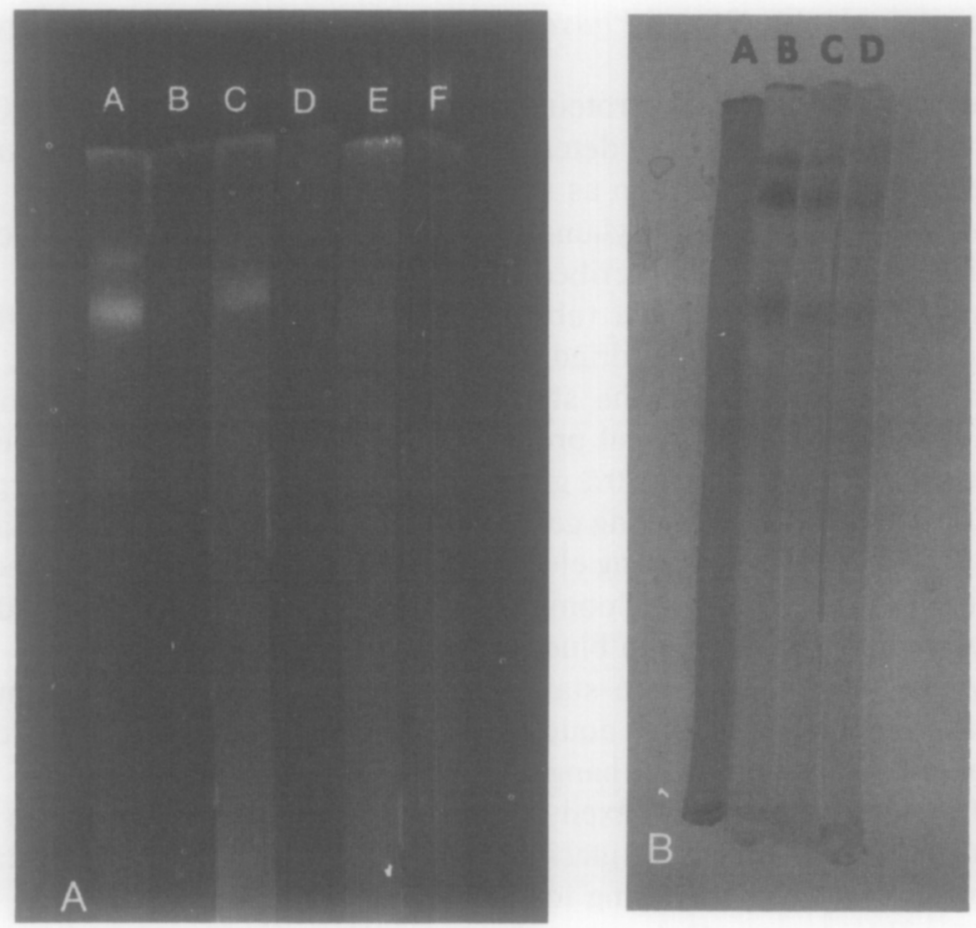

FIG. 1. (A) Fluorescent band patterns for three glycoproteins (Phaseolus lunatus lectin, Bandeiraea simplicifolia lectin, and ovalbumin, top to bottom) as a function of amount of protein and periodate oxidation. The gels contained the following amounts of each glycoprotein: Gels A and B, $2.5 \mu \mathrm{g}$; Gels C and D, $1.2 \mu \mathrm{g}$; and Gels E and F, 0.6 $\mu \mathrm{g}$. Gels $\mathrm{A}, \mathrm{C}$, and $\mathrm{E}$ were stained for carbohydrate with dansyl hydrazine as described. Control gels $B, D$, and $F$ were stained with dansyl hydrazine omitting the periodate oxidation treatment. (B) Same three glycoproteins stained with PAS or Coomassie brilliant blue. Gel A contained $2.5 \mu \mathrm{g}$ of each glycoprotein and was stained for carbohydrate with the PAS stain. Gels B, C, and D contained, respectively, $2.5,1.2$, and $0.6 \mu \mathrm{g}$ of each glycoprotein and were stained for protein with Coomassie brilliant blue.

Photography. Glycoproteins stained with dansyl hydrazine were visualized with a longwave uv transilluminator with a peak output at 366 nm (Ultraviolet Products, Inc., San Gabriel, California). Photographs were taken with a $4 \times 5$ press-type camera equipped with a Polaroid 545 film adapter. Polaroid type 57 (ASA 3200) film was used with an exposure time of $2.5 \mathrm{sec}$ at $\mathrm{f} 5.6$ with a Kodak Wratten 16 filter.

\section{RESULTS AND DISCUSSION}

A mixture of Phaseolus lunatus lectin, Bandeiraea simplicifolia lectin and ovalbumin containing, respectively, 4,6 , and $4 \%$ by weight carbohydrate was electrophoresed in $7.5 \%$ polyacrylamide gels. Decreasing 

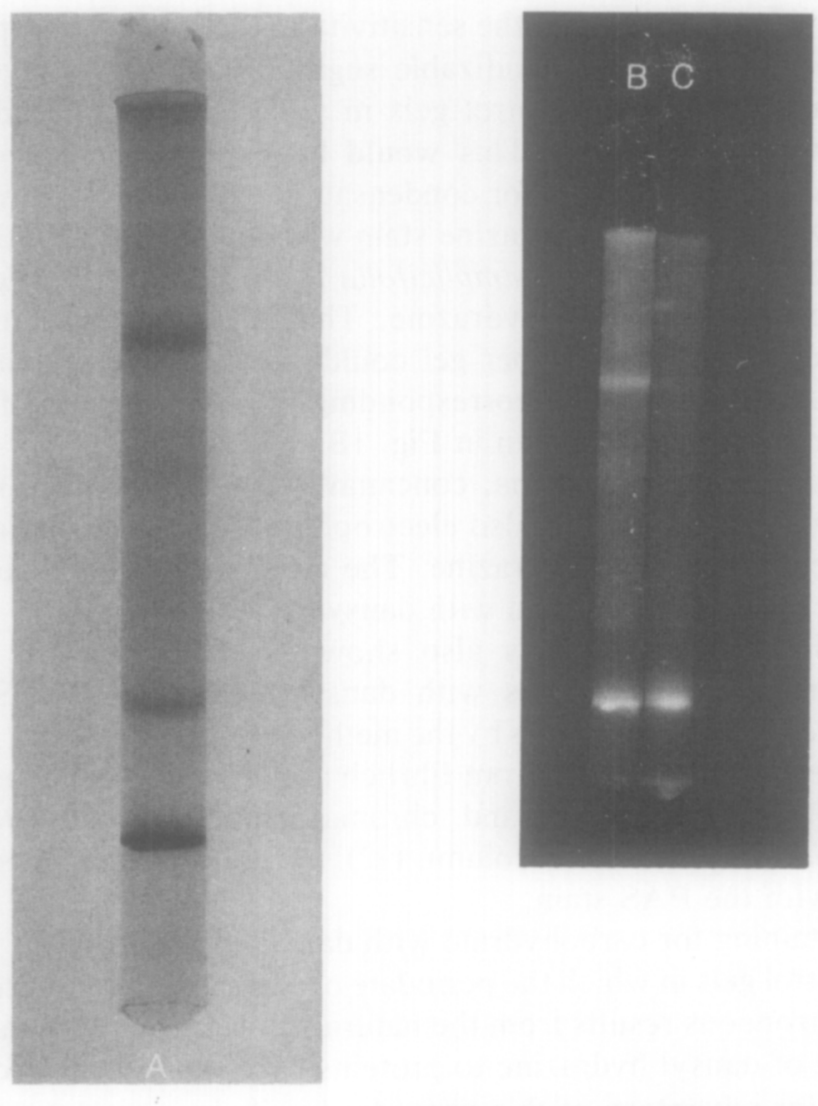

Fig. 2. Staining for carbohydrate and protein after SDS polyacrylamide gel electrophoresis of Ehrlich ascites tumor cell plasma membrane glycoproteins which bound to Bandeiraea simplicifolia-Sepharose 4B column. Glycoproteins from Ehrlich ascites tumor cell plasma membrane isolated by absorption to $B$. simplicifolia-Sepharose $4 \mathrm{~B}$ column. Each gel contained $27 \mu \mathrm{g}$ total protein. Gel A was stained for protein with Coomassie brilliant blue. Gel B was stained for carbohydrate with dansyl hydrazine as described. Gel $C$ was stained with dansyl hydrazine omitting the periodate oxidation.

amounts of each glycoprotein, $2.5,1.2$, and $0.6 \mu \mathrm{g}$, were electrophoresed and stained for sugar with dansyl hydrazine as described. Duplicate control gels were stained with dansyl hydrazine omitting the periodateoxidation step. Identical gels were also stained with Coomassie blue, PAS, or Alcian blue. The results are shown in Fig. 1A.

Bandeiraea simplicifolia lectin and Phaseolus lunatus lectin were stained intensely with dansyl hydrazine in the gel containing $2.5 \mu \mathrm{g}$ of each glycoprotein. This corresponds to approximately 150 and $100 \mathrm{ng}$ of total carbohydrate, respectively. The ovalbumin band which also contains approximately $100 \mu \mathrm{g}$ total sugar was faint. Ovalbumin contains a large number of carbohydrate residues which are resistant to periodate oxida- 
tion. This demonstrates that the sensitivity of the method is dependent on the number of periodate-oxidizable sugar residues. The glycoproteins in the dansyl hydrazine control gels in which periodate oxidation was omitted were not stained. This would be expected inasmuch as free aldehydes were not present for condensation with dansyl hydrazine. The sensitivity of the dansyl hydrazine stain was demonstrated in the fifth gel in which $0.6 \mu \mathrm{g}$ Bandeiraea simplicifolia lectin (less than $40 \mathrm{ng}$ of sugar) was detected with dansyl hydrazine. The three glycoproteins each at amounts of $2.5 \mu \mathrm{g}$ protein per gel could not be detected with PAS or Alcian blue. However, the corresponding gels were stained for protein with coomassie blue as shown in Fig. 1B.

A mixture of three proteins, concanavalin A, chymotrypsinogen and bovine serum albumin, was also electrophoresed on polyacrylamide gels and stained with dansyl hydrazine. The nonglycosylated proteins (each at $5 \mu \mathrm{g}$ per gel) failed to stain with dansyl hydrazine.

The utility of the stain is also shown in Fig. 2 which illustrates the staining of glycoproteins with dansyl hydrazine after SDS polyacrylamide gel electrophoresis by the method of Laemmli et al. (11). These glycoproteins were isolated from Ehrlich ascites tumor cell plasma membranes after solubilization and chromatography over a Bandeiraea simplicifolia-Sepharose 4B column (12). Here again no bands were observable with the PAS stain.

When staining for carbohydrate with dansyl hydrazine, it is important to run control gels in which the periodate oxidation step is omitted. This is to avoid erroneous results from the natural fluorescence of some proteins or binding of dansyl hydrazine to proteins by noncovalent forces.

The major advantage of this procedure is its increased sensitivity for the detection of glycoproteins after polyacrylamide gel electrophoresis. The staining is performed after electrophoresis to avoid changes in glycoprotein mobility which may occur if the glycoproteins were stained prior to electrophoresis. The condensation and reduction are carried out in DMSO which causes negligible dehydration and shrinkage of the gels.

Note added in proof. While this manuscript was in preparation, a procedure for introducing dansyl hydrazine as a fluorescent marker into the sialic acid residues of glycoproteins in solution was published (13).

\section{REFERENCES}

1. Segrest, J. P., and Jackson, R. L. (1972) Methods Enzymol. 28, 54.

2. Fairbanks, G., Steck, T. L., and Wallach, D. F. H. (1971) Biochemistry 10, 2626.

3. Wardi, A. H., and Michos, G. A. (1972) Anal. Biochem. 49, 607.

4. Kapitany, R. A., and Zebrowski, E. J. (1973) Anal. Biochem. 56, 361.

5. Chayen, R., Drir, R., Gould, S., Harell, A. (1971) Anal. Biochem. 42, 283.

6. Agrawal, B. B. L., and Goldstein, I. J. (1965) Biochem. J. 96, 23C.

7. Hayes, C. E., and Golstein, I. J. (1974) J. Biol. Cehm. 249, 1904. 
8. Bessler, W., and Goldstein, I. J. (1974) Arch. Biochem. Biophys, 165, 444.

9. Lowry, O. H., Rosenbrough, N. J., Farr, A. L., and Randall, R. J., (1951) J. Biol. Chem. 196, 265.

10. Reisfeld, R. A., Lewis, V. J., and Williams, D. E., (1962) Nature (London) 195, 281.

11. Laemmli, V. K. (1970) Nature (London) 227, 680.

12. Ross, T., Hayes, C. E., and Goldstein, I. J., Carbohyd. Res., in press.

13. Weber, P., and Hof, L. (1975) Biochem. Biophys. Res. Commun. 65, 1298. 